Ann. Biol. anim. Bioch. Biophys., I976, 16 (4), 545-559.

\title{
ÉTUDE DE LA FORMATION DE L'ANTRUM DANS LES FOLLICULES DE L'OVAIRE DE RATTE ET DE VACHE NORMALES OU STIMULÉES PAR PMSG
}

\author{
J. C. MARIANA et J. MACHADO \\ avec la collaboration technique de Marie José Manicom \\ Station de Physiologie de la Reproduction, \\ Centre de Recherches de Tours, I. N. R. A., \\ Nouzilly, 37380 Monnaie.
}

\begin{abstract}
RÉSUMÉ
Dans les ovaires de rattes et vaches non traitées et recevant une injection d'hormone PMSG, la formation de l'antrum dans les follicules a été précisée par des méthodes quantitatives. Les mesures de surface du follicule ou de l'antrum discret effectuées par planimétrie, de nombres de cellules ou de pourcentages de surface occupée par les espaces intercellulaires évalués par des méthodes stéréologiques donnent une indication morphologique de l'état du follicule.

Chez le Rat jusqu'à $6000 \mu^{2}$, la taille du follicule (définie par la surface de la section du follicule contenant le nucléole de l'ovocyte) augmente surtout par suite de la multiplication des cellules de la granulosa. Toutefois, à partir de 4 ooo $\mu^{2}$, on observe une augmentation et une forte variation de la surface occupée par les espaces intercellulaires dans les follicules de même taille.

A partir de $6000 \mu^{2}$, l'antrum discret apparaît et lorsqu'il représente plus de $20 \mathrm{p}$. 100 de la surface du follicule, les espaces intercellulaires constituent un pourcentage de la surface du follicule (antrum diffus) relativement constant (Io à $30 \mathrm{p}$. Iоo). Pendant la période où cet antrum discret est encore petit $(R<20 \mathrm{p}$. IOo) l'antrum diffus varie beaucoup entre follicules.

A partir de ro $000 \mu^{2}$, il existe une relation linéaire entre la taille du follicule et le paramètre $\mathrm{R}$ (75 p. Ioo de la variation étant liée à la régression). Toutefois, une part de l'augmentation de taille revient encore à la multiplication des cellules.

L'action de PMSG est d'augmenter la pente de la droite de régression existant entre la taille $\mathrm{du}$ follicule et le paramètre $\mathbf{R}$. Cette hormone fait également apparaître l'antrum discret dans des follicules de plus petite taille et accroît le nombre de follicules de plus grande taille et de rapport $R>25$ p. IOo. Ceci signifie qu'elle agit sur les deux composants de la croissance du follicule, multiplication et sécrétion des cellules de la granulosa et sur toutes catégories de follicules.

Chez la Vache, l'antrum discret débute à partir d'une taille du follicule supérieure à o,or $\mathbf{m m}^{2}$. Une relation linéaire existe également entre la taille du follicule et la surface de l'antrum occupée par le follicule pour des tailles comprises entre o, or $\mathrm{mm}^{2}$ et $4 \mathrm{~mm}^{2}$. Plus nettement que chez la Ratte, la multiplication cellulaire cesse de participer a l'accroissement de taille du follicule à partir de $4 \mathrm{~mm}^{2}$.
\end{abstract}


La courbe de distribution des tailles des follicules d'un ovaire de femelle immature est polymodale (de REVIERs, I974), ces différents modes correspondant vraisemblablement à des phases plus ou moins rapides de l'augmentation de la taille du follicule. La formation de l'antrum n'apparait pas comme un point critique dans cette courbe de distribution des tailles de follicules. Il convient donc de faire une étude particulière de la formation de l'antrum et de suivre son développement dans le follicule, pour des conditions normales de fonctionnement de l'ovaire et après une stimulation gonadotrope.

\section{MATÉRIEL ET MÉTHODES}

Pour cette étude, cinq types d'animaux ont été utilisés :

- 2 rattes normales de 3 mois abattues au protrus,

- 2 rattes de 3 mois recevant Io ou 20 UI de PMSG au début du diøstrus I et abattues au prœstrus suivant,

- I veau normal de 3 mois,

- I vache adulte cyclique abattue en début d'œstrus,

- I vache adulte recevant $\mathrm{I} 600$ UI de PMSG au $\mathrm{I}^{\mathrm{e}}$ jour du cycle et abattue également en début d'œstrus.

Chez la Ratte, les stades du cycle sont déterminés par frottis vaginaux ; les rattes sont tuées le soir du prostrus et les ovaires fixés au Bouin Hollande, inclus, coupés en série à $5 \mu$ et colorés au trichrome de Mallory.

Tous les follicules de section maximum supérieure à $2000 \mu^{2}$ ont été étudiés. Quatre critères permettant de caractériser ces follicules ont été utilisés :

- la surface de la section du follicule limitée à la lame basale et contenant le nucléole de l'ovocyte $\left(\mathbf{S}_{\mathrm{G}}\right)$,

- le nombre de cellules de la granulosa quand ce nombre ne dépasse pas 300, dans la section du follicule contenant le nucléole de l'ovocyte.

- la surface de l'antrum $\left(S_{A}\right)$ quand elle est supérieure dans la section considérée à $500 \mu^{2}$,

- l'importance des espaces limités par les cellules de la granulosa et déterminée par la méthode stéréologique (SolARI, I973).

Chez la vache adulte cyclique, l'apparition de l'œstrus est détectée matin et soir par un taureau vasectomisé.

Les ovaires immédiatement après abattage de la vache sont fixés dans le Bouin Hollande sublimé, inclus et montés sur lame à raison d'une coupe sur cinq, l'épaisseur des coupes étant Io $\mu$. La surface des follicules est mesurée par planimétrie dans les sections contenant l'ovocyte. La surface de l'antrum n'est planimétrée que lorsqu'elle est supérieure à $500 \mu^{2}$.

\section{Définitions}

Nous appellerons "Antrum discret » un antrum dont la section mesurée est supérieure à $500 \mu^{2}$ et " Antrum diffus " l'ensemble des espaces intercellulaires connexe tel qu'aucun de ces espaces n'ait localement une surface supérieure à $500 \mu^{2}$.

$\mathrm{Au}$ cours de cette étude, nous appellerons $\mathrm{R}$ le rapport entre la surface $\mathrm{S}_{\mathrm{A}}$ de l'antrum discret et la surface $S_{G}$ du follicule, ces deux surfaces ayant été mesurées sur la même section du follicule considéré ; nous insistons sur le fait qu'en aucun cas $\mathrm{R}$ ne doit être considéré comme une estimation stéréologique de la proportion de volume du follicule occupé par l'antrum ; R est seulement une indication morphologique de l'état du follicule.

Nous définirons en outre $S_{0}$ comme la surface du plus petit follicule rencontré dans l'ovaire et ayant un antrum discret. 


\section{RÉSULTATS}

I. - Croissance folliculaire dans l'ovaire de ratte normale.

Relation taille du follicule $\left(\mathbf{S}_{\mathrm{G}}\right)$ nombre de cellules et espaces intercellulaires

Jusqu'à 4 ooo $\mu^{2}$, la taille du follicule et le nombre de cellules de la granulosa croissent de façon linéaire (fig. I).

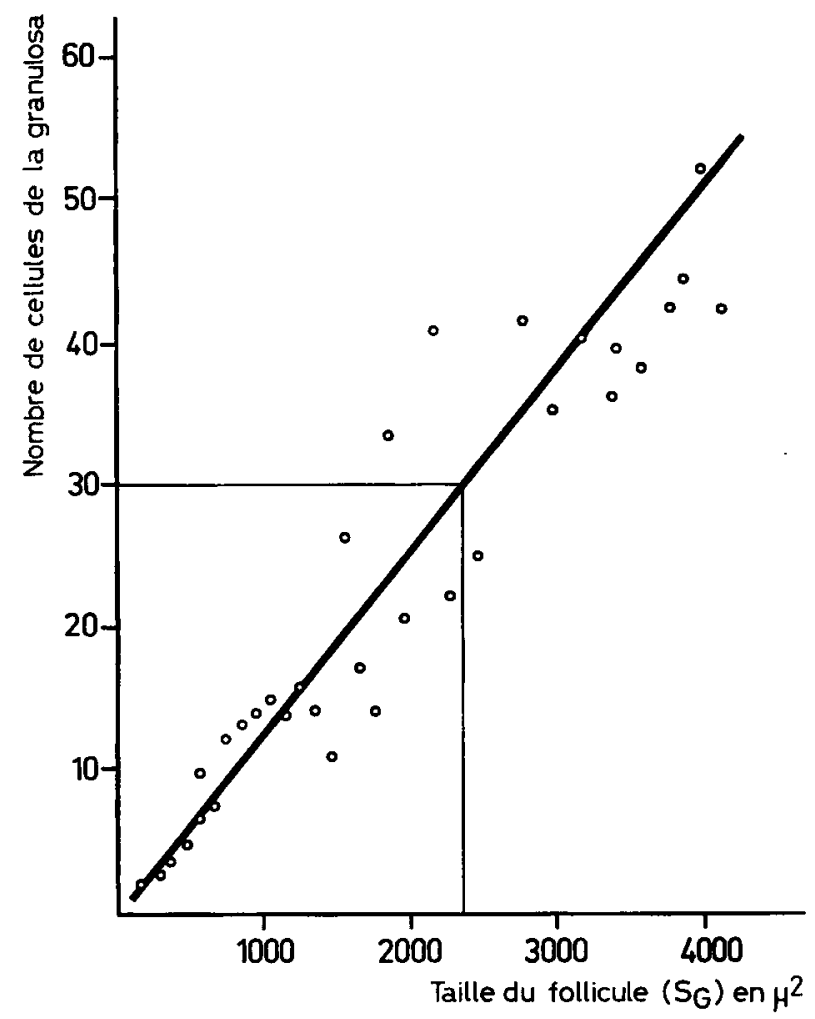

Fr. I. - Relation entre la taille du follicule et le nombre des cellules de la granulosa dans les follicules d'une taille inférieure à $4000 \mu^{2}$ d'ovaire de ratte adulte tuée au proestrus

La pente de la droite de régression entre $S_{G}$ et le nombre de cellules de la granulosa est de 0,012 .

Au-delà de $4000 \mu^{2}$ (fig. 2), cette tendance linéaire est maintenue bien que la dispersion du nombre des cellules pour une surface donnée ou de la surface pour un nombre de cellules donné semble augmenter ; ce phénomène de dispersion croissante est net pour une taille de follicule supérieure à ro ooo $\mu^{2}$.

Il est clair que l'augmentation de la taille des follicules est liée à la multiplication des cellules mais qu' utn autre phénomène s'y superpose pour en accroître la dispersion ; 


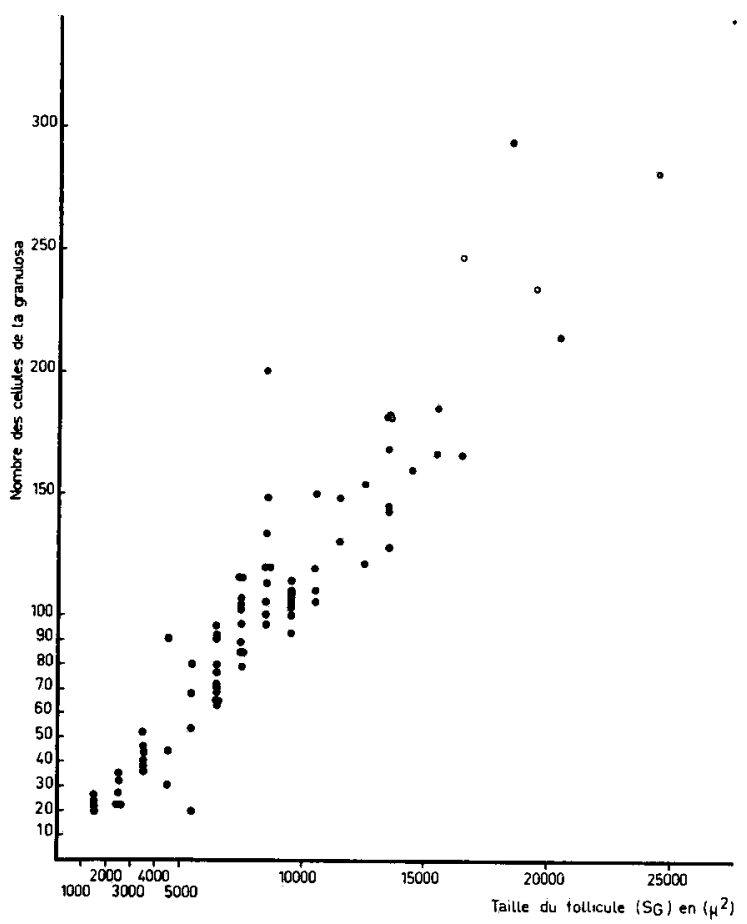

FIG. 2. - Relation entre la taille du follicule et le nombre des cellules de la granulosa dans les follicules d'une taille supérieure à $4000 \mu^{2}$ d'ovaire de ratte adulte tuée au proestrus

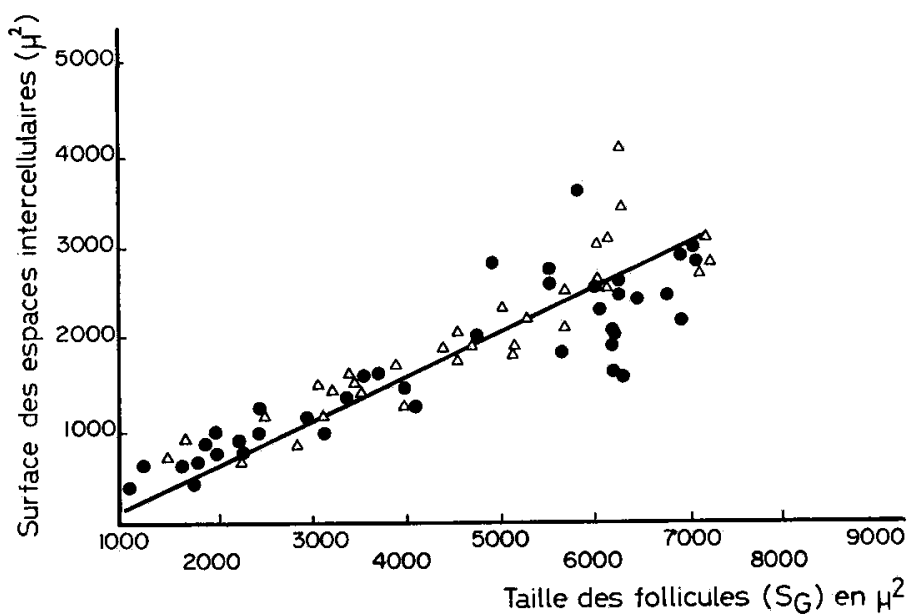

FIG. 3. - Relation entre la surface occupée par les espaces intercellulaires et la surface totale d'une section de follicule ovarien de ratte adulte tuée au prostrus (les deux signes représentent deux animaux distincts) 
en effet, on observe que l'accroissement des espaces intercellulaires est lié à celui du follicule (fig. 3) ; la pente de la droite de régression entre $S_{G}$ et la surface occupée par les espaces intercellulaires est 0,50 .

Au-delà d'une taille supérieure à $6000 \mu^{2}$, cette dernière relation devient à son tour moins stricte ; en effet, pour cette dernière valeur de $S_{\mathfrak{G}}$, l'antrum discret apparaît.

\section{II. - Formation et croissance de l'antrum discret chez la ratte normale}

I. L'accroissement des espaces intercellulaires est proportionnel à celui de la surface du follicule ; en effet, la droite de régression entre la surface des espaces intercellulaires et $S_{G}$ passe près de l'origine (fig. 3). Cet accroissement est cependant variable d'un follicule à un autre de même taille au début de la formation de l'antrum discret ; la proportion de la surface du follicule occupée par les espaces intercellulaires, c'est-à-dire le pourcentage d'antrum diffus, varie entre Io et 70 p. Ioo (fig. 4). Quand le rapport $(R)$ entre la surface de l'antrum discret $\left(\mathbf{S}_{A}\right)$ et la surface du follicule $\left(\mathbf{S}_{G}\right)$ atteint $20 \mathrm{p}$. IOO, la proportion précédente varie entre Io et $3^{\circ} \mathrm{p}$. IOO.

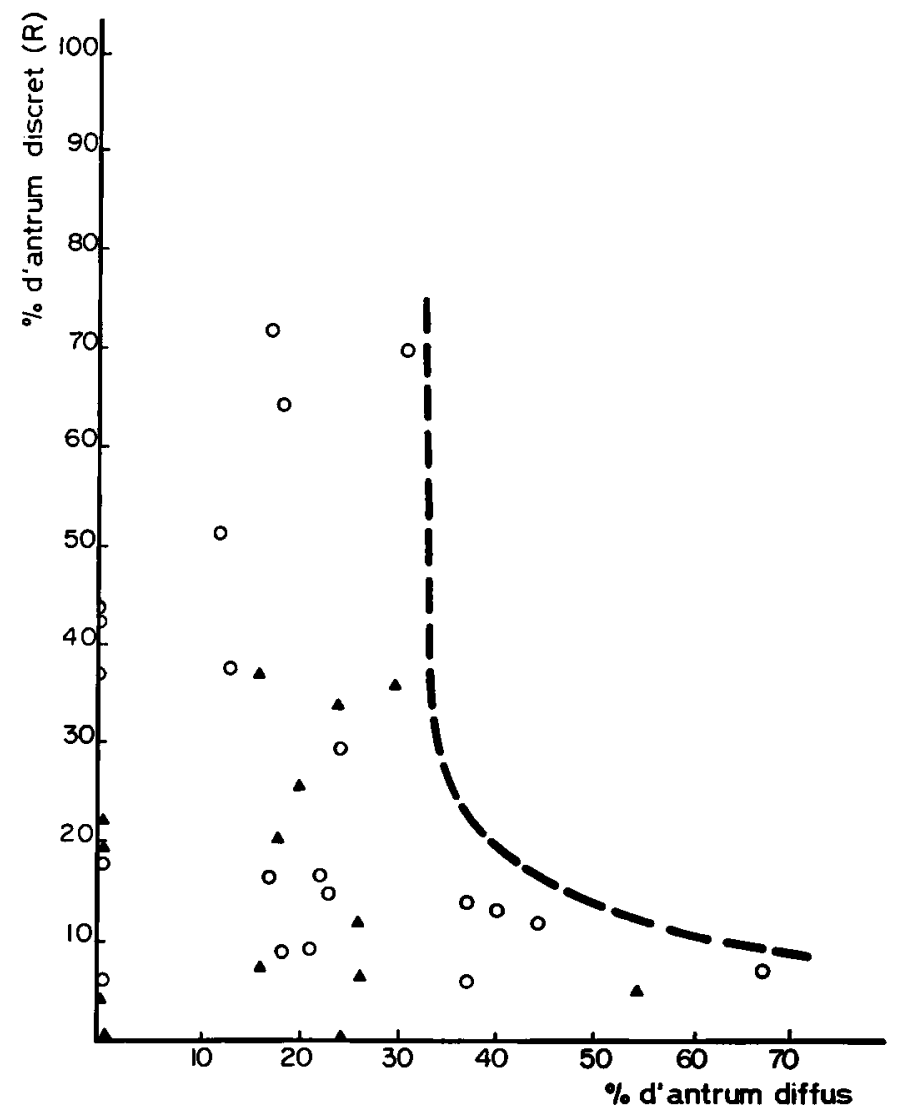

Fig. 4. - Variations du pourcentage d'antrum discret en fonction de la proportion de la surface occupée par l'antrum diffus dans le follicule ovarien de rattes adultes tués au procestrus (les deux signes représentent des animaux différents). 

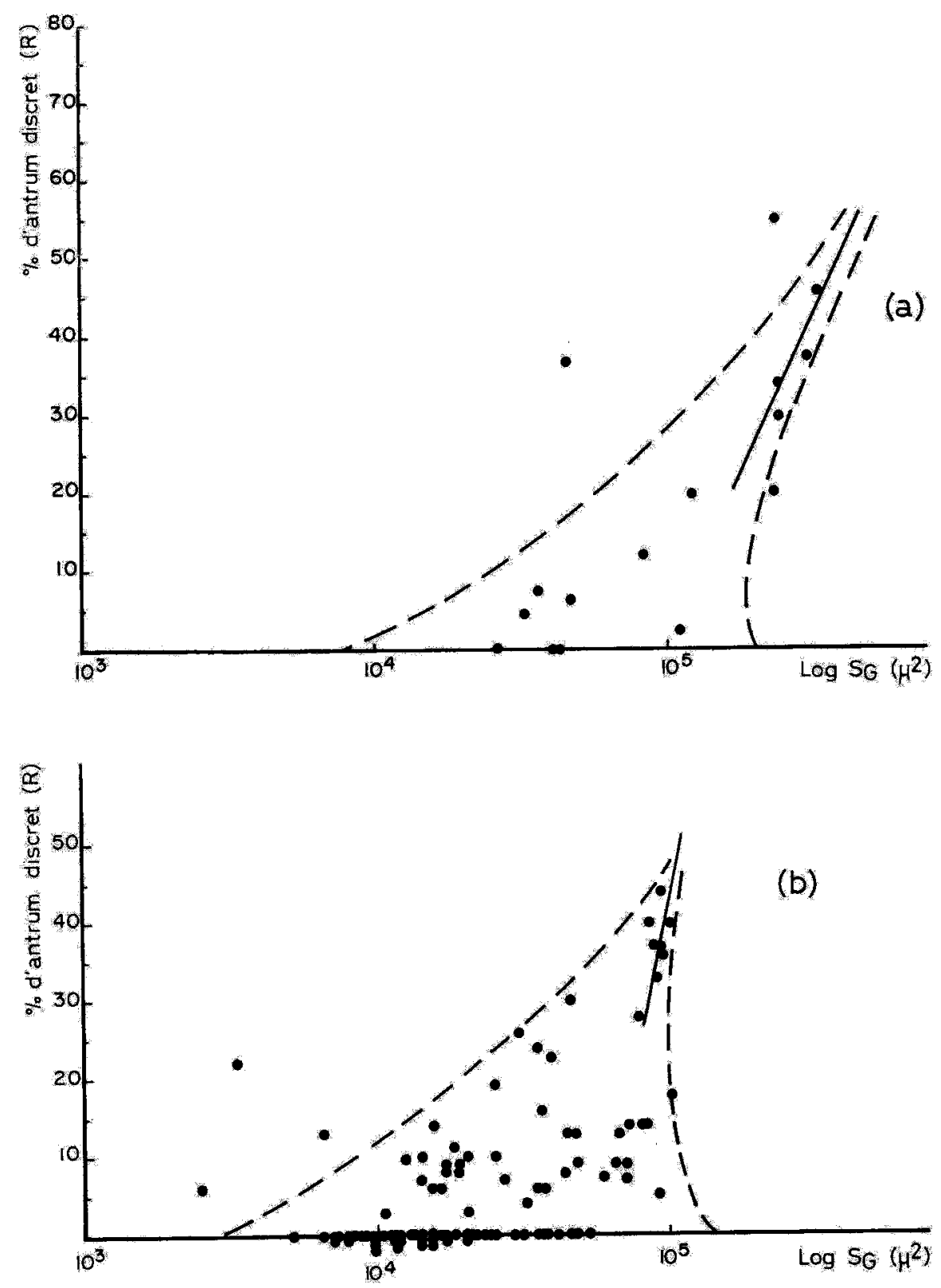

FrG 5 


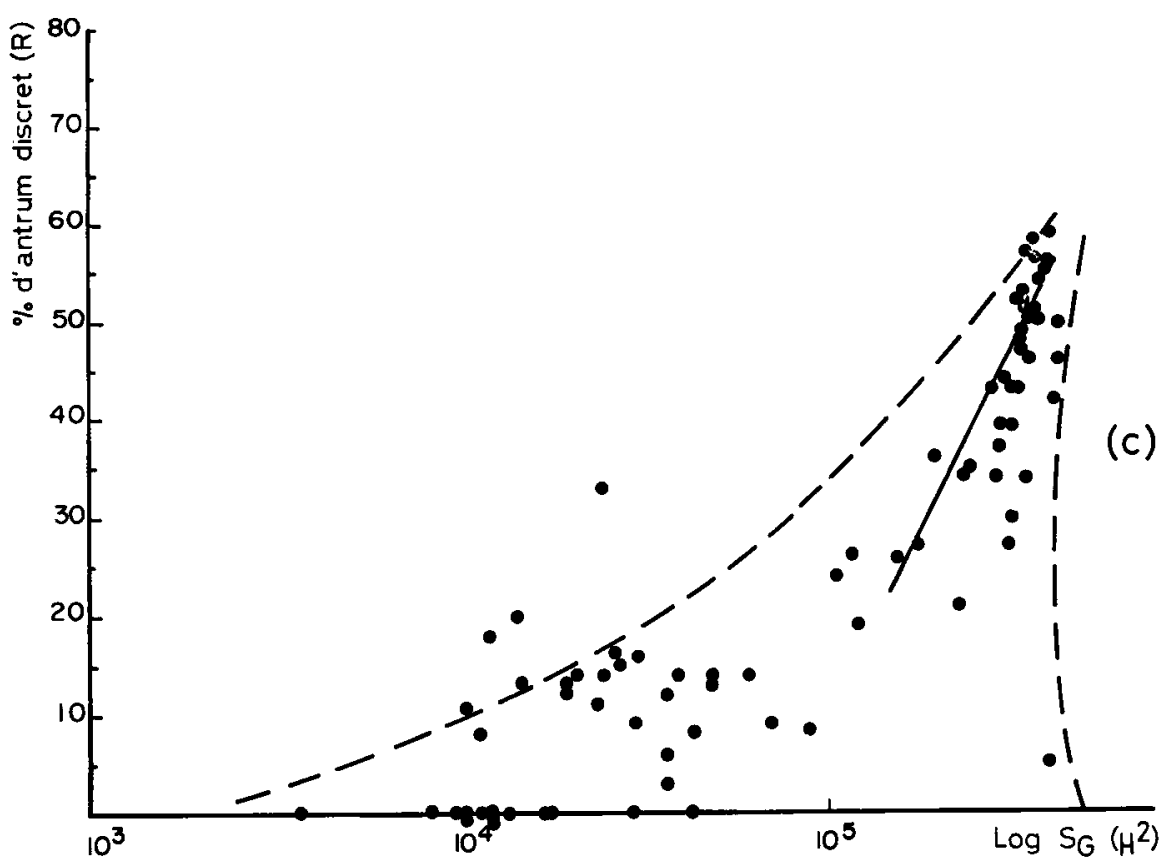

Fig. 5. - Relation entre le log. de la taille du follicule $\left(\mathrm{S}_{\mathbf{G}}\right)$ et la proportion de la surface occupée par l'antrum discret, dans l'ovaire de ratte adulte tuée au prostrus (A), dans l'ovaire de ratte adulte injectée de 10 UI de PMSG (B) ou de 20 UI (C) au metcestrus et tuées au prostrus.

2. Lorsque la taille du follicule est supérieure à $10^{5} \mu^{2}$ (fig. $5 \mathrm{~A}$ ), $\mathrm{R}$ croît rapidement et passe de 25 à $60 \mathrm{p}$. Ioo ; la pente de la droite de régression correspondante que l'on peut ajuster entre $R$ et $\log$. $S_{G}$ varie d'un animal à l'autre, entre 40 et 80 .

Ainsi, pour le témoin figurant sur le graphique $5 a$, la pente de la droite de régression est $79,57\left(R=79,57 \log\right.$. $\left.S_{G}+396,26\right)$; on peut estimer à $74 \mathrm{p}$. Ioo la part de la variation liée à la régression elle-même et à 26 p. Ioo celle due aux variations aléatoires. Pour des follicules de taille identique et inférieure à $10^{5} \mu^{2}, R$ peut varier du simple au triple, mais il ne semble pas exister de tendance à une relation entre les deux paramètres précédents.

L'examen de la figure 6 A confirme que l'accroissement de volume des gros follicules par diffusion de liquide folliculaire est certainement un phénomène rapide; en effet, on peut distinguer deux groupes de follicules (I et II) : ceux dont la taille est comprise entre 2000 et $I 803 I 6 \mu^{2}$ avec $R<25 \mathrm{p}$. Ioo (I) et ceux dont la taille est supérieure à $\mathrm{I} 803 \mathrm{I} 6 \mu^{2}$ et $\mathrm{R}>25 \mathrm{p}$. IO0 (II). Cette classification n'exclut pas que l'on trouve des follicules avec $R<25$ p. Ioo dans la seconde catégorie et des follicules avec $R>25 \mathrm{p}$. Ioo dans la première.

Il n'est pas possible, au vu de ces résultats, de dire quelle part de la croissance du follicule revient à l'augmentation de la taille de l'antrum discret et quelle part revient à la multiplication cellulaire; on notera cependant que les plus gros follicules $\left(\mathrm{S}_{\mathrm{G}}>I \mathrm{O}^{5} \mu^{2}\right.$ et $\mathrm{R}>25 \mathrm{p}$. IOo) ont un nombre de cellules supérieur à celui des follicules de taille inférieure à $\mathrm{IO}^{5} \mu^{2}$ (fig. $6 \mathrm{~A}$ ) c'est-à-dire qu'un accroissement du nombre de cellules de la granulosa se produit lorsque le follicule augmente de taille. 
Follicules ayant R) $25 \%$

Follicules ayant $10 \%<R<25 \%$

Nombre de follicules

$\square$ Follicules ayant $\mathrm{R}<10 \%$

ayant $R$ ) $25 \%$

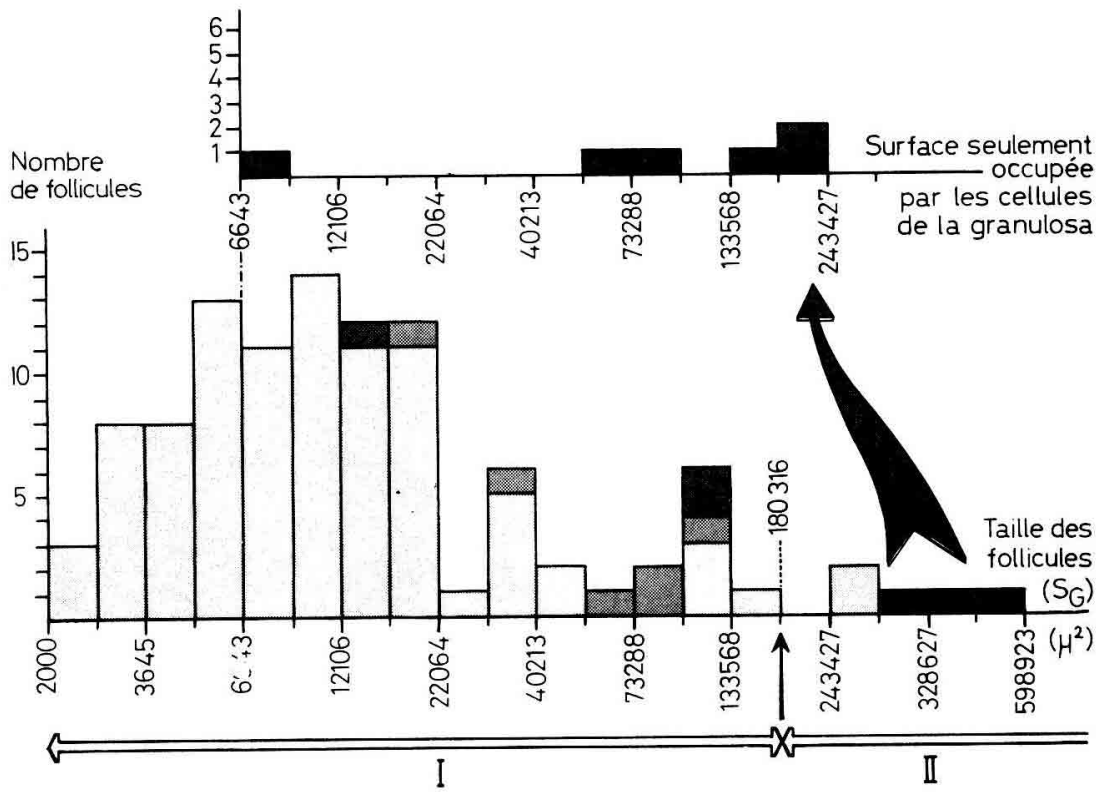

FIG. $6 \mathrm{~B}$ : Ratte traitée au métcestrus avec 10 UI de PMS et tuèe au prostrus

Follicules ayant R> $25 \%$

Nombre de follicules

Follicules ayant $10 \%<R<25 \%$ ayant $R>25 \%$

Follicules ayant R $<10 \%$

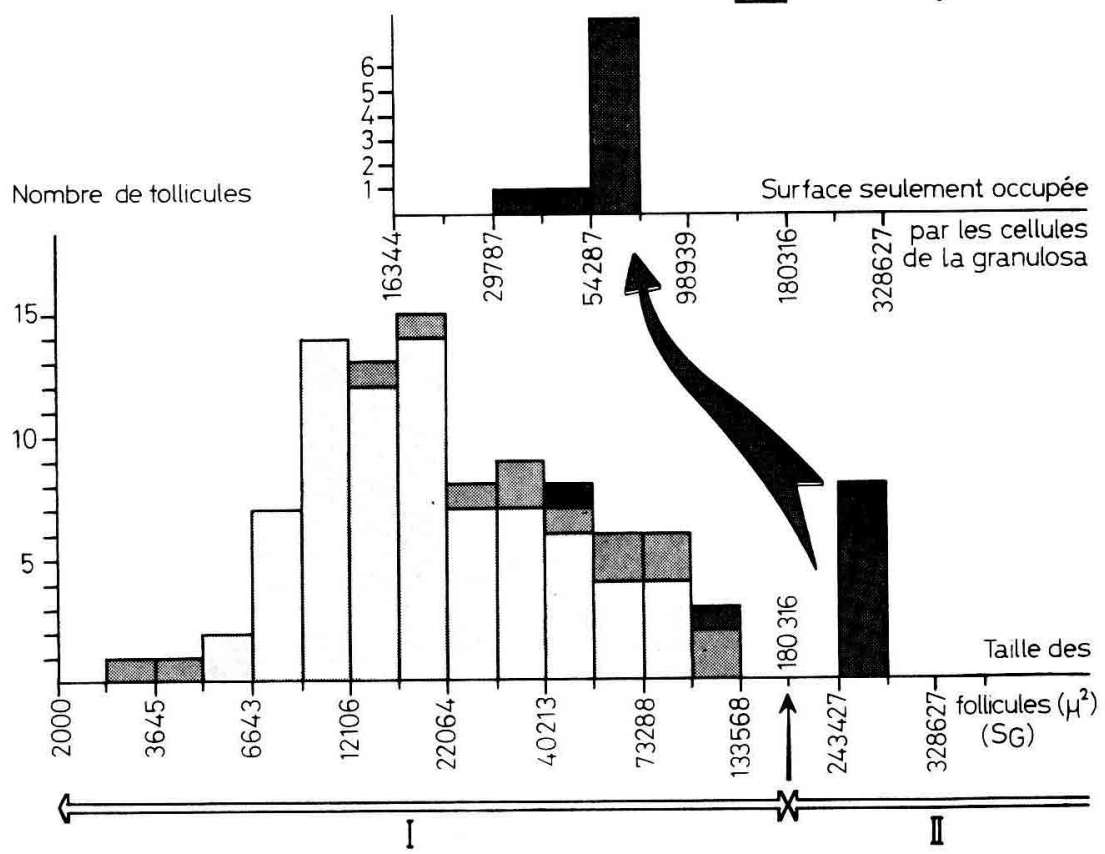


FIG. $6 \mathrm{C}:$ Ratte traitée au métoestrus avec 20 CI de PMSG et tuée au prostrus

Follicules ayant R $>25 \%$

Follicules ayant $10 \%<R<25 \%$

Follicules ayant $R<10 \%$

Nombre de follicules

ayant R $>25 \%$

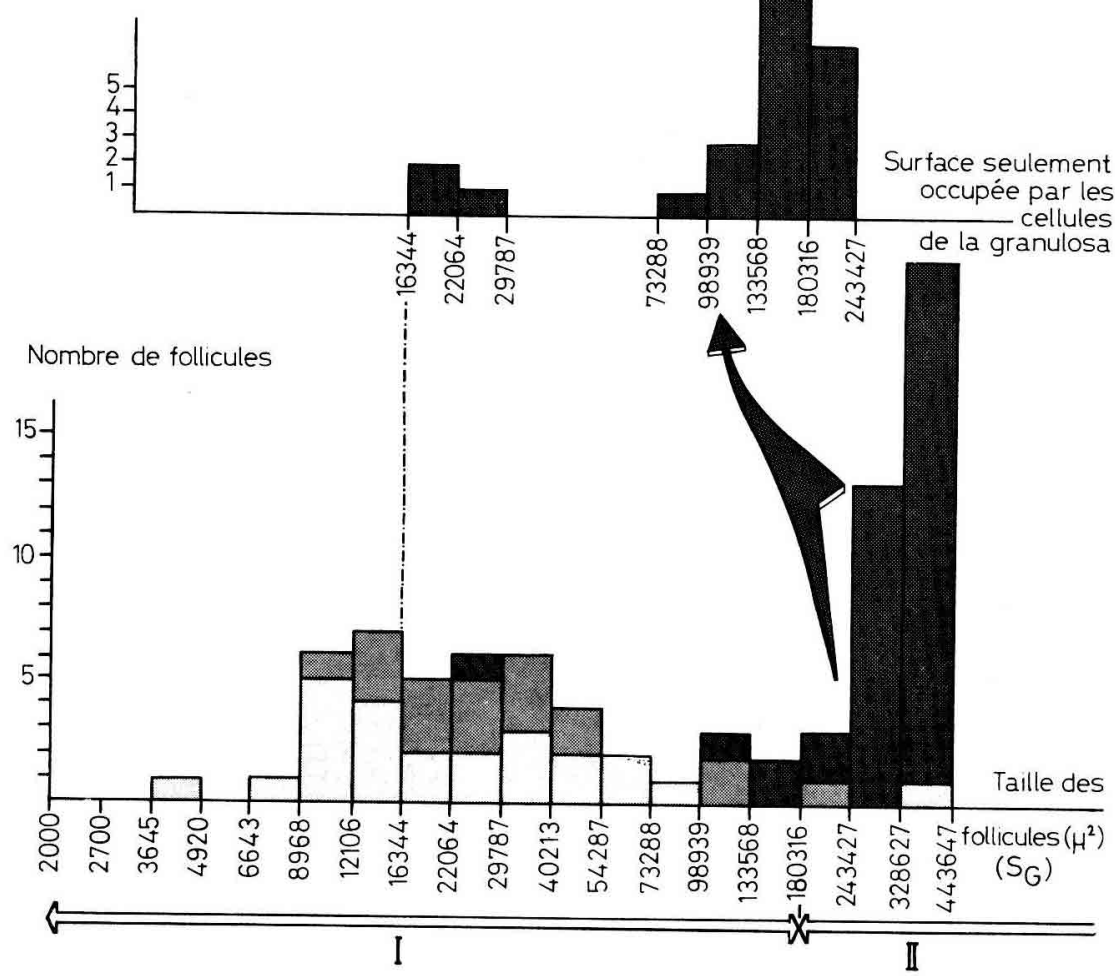

líg. 6. - Courbes de fréquence des populations folliculaires d'ovaire de rattes adultes tuées au prostrus non traitées (A), ou injectées de $10 \ell^{*} I$ (B) ou de 20 UI (C). Les follicules sont classés selon leur taille et la proportion de la surface occupée par l'antrum discret est également représentée (coordonnées semiJogarithmiques). 


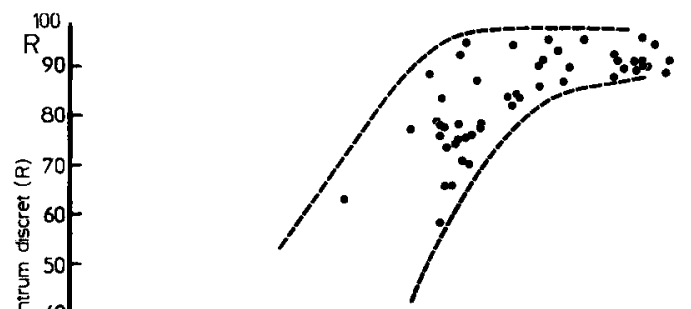

A. VACHE ADULTE TEMONN TUEE A LOOESTRUS
(SANS TRAITEMENT)

:

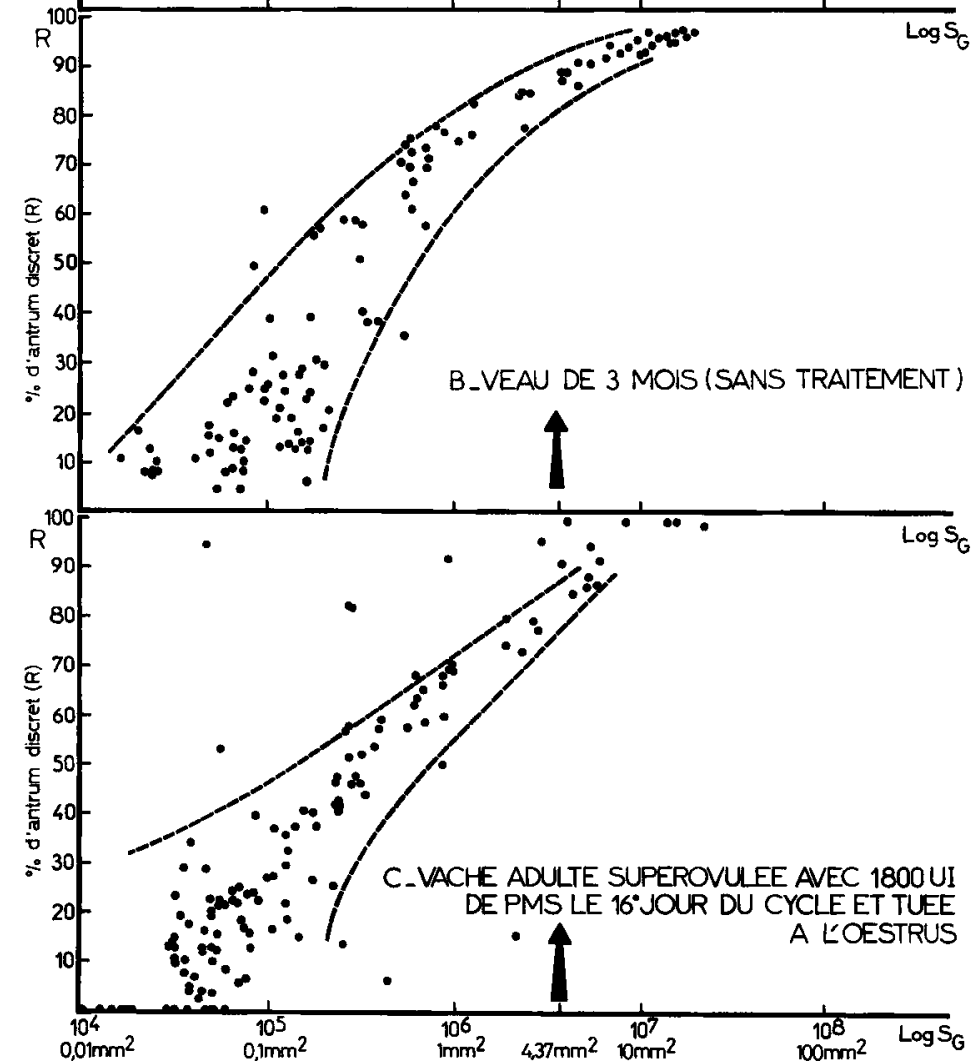

FIG. 7. - Relation entre le logarithme de la taille du follicule $\left(\mathrm{S}_{\mathrm{G}}\right)$ et la proportion de la surface occupée par l'antrum discret (R) dans l'ovaire de vache adulte abattue à l'cestrus et non traitée (A) ou traitée avec PMSG (C) ou de génisse de 3 mois (B). 

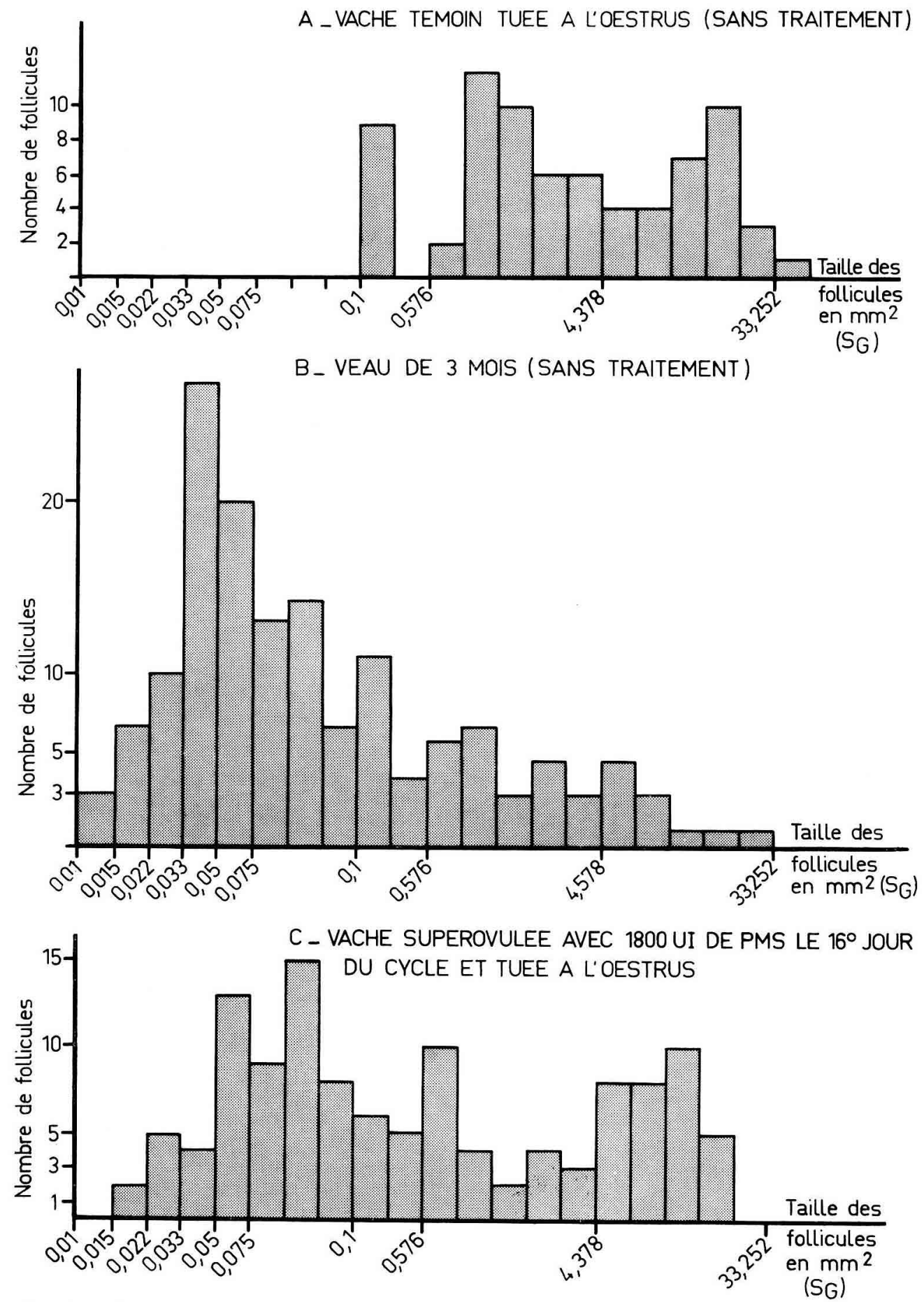

'rik. '. - Couroe àe fréquence ảe follicules classés selon leur taille (coordonnées semi-logarithmiques) d'ovaires de vaches abattues à l'astrus non traitées (A), ou recevant une injection de PMSG (C) ou de genisises de 3 mois (B). 


\section{III. - Action de PMSG sur la croissance du follicule de l'ovaire de rat : multiplication cellulaire et développement de l'antrum}

Nous n'avons pas de résultat détaillé sur la distribution des espaces intercellulaires mais il ne nous semble pas qu'il y ait de différence entre la ratte témoin et la ratte superovulée sur ce point.

On constate que l'antrum discret (c'est-à-dire lorsque $\mathrm{R}>$ Io p. IOO) apparaît plus précocement chez la ratte traitée que chez la ratte témoin; en effet, la taille des follicules de ratte traitée avec 20 UI où l'antrum apparait est environ $8968 \mu^{2}$ alors que cela se produit pour une taille des follicules de I 2 I06 $\mu^{2}$ chez les rattes témoins. Ce phénomène est par ailleurs plus net chez la ratte ayant reçu 20 UI que chez celle n'ayant reçu que ro UI (fig. $6 \mathrm{~B}, \mathrm{C}$ ).

$L_{a}$ pente de la droite de régression entre $R$ et $\log$. $S_{G}$ est plus élevée chez les rattes traitées par PMSG que chez les témoins (fig. $5 \mathrm{~B}, \mathrm{C}$ ).

\section{IV. - Croissance folliculaire de l'ovaire de vache}

La formation de l'antrum discret a été étudiée chez une vache témoin adulte non traitée pour des follicules dont la taille est supérieure à $\mathrm{o}, \mathrm{I} \mathrm{mm}^{2}$ et chez le veau normal et la vache superovulée pour des follicules dont la taille est supérieure à 0,0 I $\mathrm{mm}^{2}$.

Chez ces trois types d'animaux et particulièrement chez le veau, on peut éga- lement mettre en évidence une relation linéaire entre $\log$. $S_{G}$ et $R$ pour les follicules dont la taille est comprise entre o,or $\mathrm{mm}^{2}$ et $4 \mathrm{~mm}^{2}$. La croissance des follicules se fait surtout par augmentation de la surface de l'antrum discret et de moins en moins par multiplication cellulaire (fig. $7 \mathrm{~A}, \mathrm{~B}, \mathrm{C}$ ). On peut calculer par exemple, pour un follicule de taille supérieure à $2 \mathrm{~mm}^{2}$ et inférieure à $4,37 \mathrm{~mm}^{2}$ que la multiplication cellulaire ne rend compte que de moins de 2 p. 100 de l'accroissement de la taille. Pour des follicules de taille supérieure à $4,37 \mathrm{~mm}^{2}$, le volume du follicule n'augmente plus que par pénétration de liquide folliculaire ; l'épaisseur de la granulosa n'augmente plus et diminue même; $\mathbf{R}$ atteint une valeur limite de façon progressive chez la vache non traitée (fig. $7 \mathrm{C}$ ).

Comparées au veau de 3 mois, les vaches normales et superovulées ont un nombre de follicules d'une taille supérieure à $4,37 \mathrm{~mm}^{2}$ (fig. $8 \mathrm{~A}, \mathrm{~B}, \mathrm{C}$ ) relativement plus important ; ces follicules constituent le stock préovulatoire et leur volume croît rapidement au moment du prostrus. Remarquons que pour les deux vaches adultes normale et superovulée étudiées ici, la différence entre le nombre des follicules préovulatoires est à peine notable.

\section{DISCUSSION}

Dans un précédent article (MARIANA, I972), nous avons essayé de caractériser les étapes de la croissance folliculaire par un certain nombre de paramètres tels que le nombre des cellules de la granulosa et leur répartition autour de l'ovocyte. 
Les résultats rapportés ici confirment que la croissance des follicules n'est pas réductible à une multiplication des cellules de la granulosa, particulièrement pour les follicules dont la taille dépasse $6000 \mu^{2}$ chez la Ratte. On n'a montré l'importance de la diffusion rapide du liquide folliculaire, chez la Ratte en fin de prostrus, normale ou stimulée par l'hormone PMSG (une des doses injectées - 20 UI - induisant le maximum d'ovulations chez les rattes de cette souche : MARIANA, 1974) chez le veau immature non traité ou chez la vache adulte normale ou superovulée par PMSG.

Les espaces intercellulaires augmentent et permettent très précocement la diffusion des fluides dans la granulosa; la phase préovulatoire correspond à une accélération de la pénétration du liquide folliculaire pour des follicules ayant un nombre de cellules dans la granulosa maximum par rapport à celui des autres follicules de l'ovaire.

Chez les rattes en fin de prostrus, normales et superovulées, pour l'ensemble des classes de follicules de taille inférieure à $10^{5} \mu^{2}$, la proportion (R) de volume du follicule occupé par l'antrum est très variable. Elle peut varier du simple au triple pour des follicules ayant une taille donnée. Une partie de cette variabilité observée provient de l'estimation de $\mathbf{R}$, mais cette remarque n'explique pas que l'on puisse observer des follicules dont la taille est celle d'un follicule préovulatoire normal et où, cependant, il n'y a pas d'antrum discret.

Pour les follicules de taille supérieure à $10^{5} \mu^{2}$, il existe une relation entre la taille $\mathrm{du}$ follicule et la proportion de surface occupée par l'antrum; cette relation absorbe d'ailleurs l'essentiel de la variation de $\mathrm{R}$ bien que, là encore, une source certaine de variation soit liée à l'échantillonnage des surfaces. La forte pente de la droite de régression que l'on peut ajuster, illustre la pénétration importante du liquide folliculaire dans des follicules dont le volume de granulosa est déjà voisin.

L'évolution rapide de ces follicules coincide vraisemblablement avec la séquence des événements endocriniens précédant l'ovulation (NoRman et GREENwALD, I972).

On constate également que, par rapport à des rattes non traitées examinées au prœstrus, PMSG accroît le nombre des follicules normaux de très grande taille supérieure à $\mathrm{I} 80 \mathrm{o} 3 \mathrm{I} 6 \mu^{2}$; ce fait résulte probablement de la conjonction de deux phénomènes : une réduction du taux d'atrésie et une accélération du développement de l'antrum de follicules de taille inférieure à $1803 \mathrm{I} 6 \mu^{2}$ et supérieure à $2000 \mu^{2}$.

Il semble que, chez les animaux traités par PMSG, l'antrum discret apparaisse dans les follicules de taille inférieure à celle où il apparaît normalement.

Chez la Vache ou le Veau, la forme de la relation entre les deux critères $\log . \frac{S_{G}}{S_{0}}$ et le rapport $R$ est différente de celle du Rat ( $S_{G}$ surface totale du follicule, $S_{0}$ surface du plus petit follicule dans lequel débute la formation de l'antrum). L'échelle logarithmique utilisée pour les surfaces permet en effet de comparer la croissance folliculaire chez la Vache et le Rat.

La formation de l'antrum est encore plus variable chez la Vache que chez la Ratte mais, à partir d'une taille supérieure à $0,1 \mathrm{~mm}^{2}$, la pénétration de liquide folliculaire est régulièrement croissante ; en valeur relative, cette taille est comparable à celle d'un follicule en début de formation d'antrum chez la Ratte, c'est-à-dire de $6000 \mu^{2}$. Le phénomène de pénétration du liquide folliculaire chez la Ratte n'est rapide qu'au moment de l'œstrus et pour des follicules ayant déjà une certaine taille, 
mais $R$ n'atteint jamais chez le Rat les valeurs élevées obtenues dans le follicule préovulatoire de Vache.

Chez la Ratte, la contribution des multiplications cellulaires à l'augmentation du volume des gros follicules semble maintenue jusqu'au moment de l'ovulation; chez la Vache, cette même contribution semble diminuer progressivement et même s'annuler avant l'ovulation des plus gros follicules $\left(S_{\mathrm{G}}: 4,37 \mathrm{~mm}^{2}\right)$.

Cet exemple de morphogenèse comparée illustre à notre avis la difficulté d'analyse de la croissance folliculaire et de la définition fonctionnelle des follicules. On peut se poser la question de savoir quels sont les follicules qui participent à la synthèse des stéroïdes dans l'ovaire alors qu'aucune solution de continuité morphologique n'est apparente. Il est vraisemblable que le critère de la taille des follicules est insuffisant, mais qu'à partir d'un stade critique de son évolution morphologique, le follicule devient sécrétoire de façon active : ce stade critique serait caractérisé par l'ensemble des valeurs des variables d'une fonction, réellement représentative de la sécrétion (SchwarTz, I969).

Nous pensons que le volume de la masse cellulaire du follicule et le volume des espaces intercellulaires sont au moins deux de ces variables et qu'une mauvaise programmation de la croissance, donc des relations entre ces paramètres, entraîne l'atrésie du follicule.

II est aussi connu depuis BulLough (I942) qu'il existe des gradients dans la formation de l'antrum et dans l'activité mitotique de la granulosa. L'investigation des multiplications cellulaires par les précurseurs radioactifs de l'ADN semble confirmer ce résultat et une hypothèse possible est que le programmateur de la croissance et de l'organisation du follicule est l'ovocyte lui-même. Le codage de cette régulation varierait d'une espèce à l'autre, bien que nous n'excluons pas l'hypothèse que la position des follicules par rapport à la vascularisation de l'ovaire soit déterminante.

Rę̧u pour publication en novembre 1975.

SUMMARY

FOLIICULAR ANTRUM FORMATION IN OVARIES

OF NORMAI, OR PMSG-TREATED RAT AND COW

The formation of the follicular antrum in normal or PMSG-treated rat and cow ovaries is studied using quantitative methods. The morphological state of the follicle is determined by planimetric measurement of the follicle or discrete antrum surface ; the number of cells or surface percentage occupied by intercellular spaces is evaluated by stereological methods.

Follicle size in rat (defined by the surface of follicle section containing the oocyte nucleolus) increases up to $6000 \mu^{2}$, principally due to granulosa, cell multiplication. However, the intercellular spaces of follicles greater than 4 ooo $\mu^{2}$ represent a very variable proportion of the surface.

The discrete antrum appears in follicles larger than $6000 \mu^{2}$. When it represents more than 20 p. 100 of the follicle surface, the intercellular spaces (diffuse antrum) constitute a relatively constant percentage of that surface ( 10 to $30 \mathrm{p}$. 100). There is wide variation between diffuse follicular antra when the parameter $R$ (the proportion of the follicular surface occupied by the discrete antrum) is smaller than $20 \mathrm{p}$. roo.

A linear relation between follicle size $\left(S_{G}\right)$ and $R$ is observed when $S_{G}>10000 \mu^{2}$ (75 p. roo of the variation is due to regression). However, some of the size increase is to be attributed to cell multiplication. 
PMSG hormone acts on this regression slope and causes the discrete antrum to appear in follicles smaller than the controls. It also increases the number of the largest follicles with $R>25$ p. roo. This hormone thus acts on the two components of follicular growth, secretion and granulosa cell multiplication, and on the whole population of different size follicles.

The discrete antrum in cow appears when follicle size is greater than o.or $\mathrm{mm}^{2}$. There is also linear relation between $S_{G}$ and $R$ in follicles ranging between $0.01 \mathrm{~mm}^{2}$ and $4 \mathrm{~mm}^{2}$. Cell multiplication no longer plays a role in size increase of follicles larger than $4 \mathrm{~mm}^{2}$. This observation is less clear in rat. $4 \mathrm{~mm}^{2}$ follicles represent the preovulatory follicle stock in treated or untreated cows during prostrus. The number of these follicles is very low in prepuberal heifers.

It is hypothesized that an harmonious development of the two components - cell mass and antrum percentage (discrete or diffuse) - is necessary if atresia is to be avoided.

\section{RÉFÉRENCES BIBLIOGRAPHIQUES}

Bellough W. S., 1942-I944. The method of growth of the follicle and corpus luteum in the mouse ovary. J. Endocr., 3, I50-155.

Mariana J. C., I972. Classification des follicules ovariens : principes et méthodes d'étude. Ann. Biol. anim. Bioch. Biophys., 12, 377-382.

Mariana J. C., r974. Êtude du nombre d'ovulations après superovulation induite par PMSG chez trois souches de rattes adultes. Ann. Biol. anim. Bioch. Biophys., 14, 623-632.

Norman R. L., Greenwald G. S., 1972. Follicular histology and physiological correlates in the preovulatory hamster. Anat. Rec., 173, 95-108.

de Reviers M. M., I974. Étude quantitative de l'action des hormones gonadotropes hypophysaires sur la population folliculaire de l'ovaire de ratte immature. Signification biologique du dosage de l'hormone folliculo-stimulante par le test de Steelman et Pohley. Thèse Doct. Univ., Tours.

Schwartz N. B., 1969. A model for the regulation of ovulation in the rat. Rec. Progr. Horm. Res., 25, I-55.

Solari A., I973. Étude quantitative d'organes et de tissus. I. Méthodes d'estimation des volumes. $A n n$ Biol. anim. Bioch. Biophys., 13, 247-265. 